\title{
Pharmacological inhibition of quorum sensing for the treatment of chronic bacterial infections
}

\author{
Morten Hentzer and Michael Givskov \\ Center for Biomedical Microbiology, BioCentrum-DTU, Technical University of Denmark (DTU), Lyngby, Denmark
}

\begin{abstract}
Traditional treatment of infectious diseases is based on compounds that aim to kill or inhibit bacterial growth. A major concern with this approach is the frequently observed development of resistance to antimicrobial compounds. The discovery of bacterial-communication systems (quorum-sensing systems), which orchestrate important temporal events during the infection process, has afforded a novel opportunity to ameliorate bacterial infection by means other than growth inhibition. Compounds able to override bacterial signaling are present in nature. Herein we discuss the known signaling mechanisms and potential antipathogenic drugs that specifically target quorum-sensing systems in a manner unlikely to pose a selective pressure for the development of resistant mutants.
\end{abstract}

J. Clin. Invest. 112:1300-1307 (2003). doi:10.1172/JCI200320074.

One of the greatest accomplishments of modern medicine has been the development of antimicrobial pharmaceuticals for the treatment of infectious diseases. Alexander Fleming discovered the first antibiotic, penicillin, in 1928 , and after over half a century of intense research, most acute bacterial infections can be treated effectively with antibiotics. Conventional antibiotics possess broadrange efficacy via toxic or growth-inhibitory effects on target organisms. However, an increased frequency of bacterial mutations has resulted in a significantly increased incidence of antibiotic resistance. The horizontal spread of resistance genes to other bacteria of the same or different species has been shown to rapidly create bacterial populations with (a) an increased ability to degrade antibacterial compounds; (b) decreased permeability; (c) decreased affinity for the antibiotic; or, finally, (d) increased efflux of many different antibiotics $(1,2)$. The increasing occurrence of multiresistant pathogenic bacterial strains has gradually rendered traditional antimicrobial treatment ineffective. Today, a global concern has emerged that we are entering a post-antibiotic era with a reduced capability to combat microbes, and, hence, the development of novel therapeutic approaches to the treatment of bacterial infections constitutes a focal point of modern research. The alternative to

\footnotetext{
Address correspondence to: Michael Givskov, Center for Biomedical Microbiology, BioCentrum, Building 301, Technical University of Denmark, DK-2800 Lyngby, Denmark. Phone: 45-45252769, or 45-21409867; Fax: 45-45932809; E-mail:immg@pop.dtu.dk.

Conflict of interest: Michael Givskov is the founder and vice president of QSI Pharma A/S (Lyngby, Denmark).

Nonstandard abbreviations used: $N$-acyl-homoserine lactone (AHL); exopolymeric substance (EPS);

autoinducer 2 (AI-2); $S$-adenosyl methionine (SAM);

Pseudomonas quinolone signal (PQS).
}

antibiotic-mediated bacteria killing or growth inhibition is attenuation of bacterial virulence such that the organism fails to establish successful infection and, in consequence, is cleared by the host immune response. Compounds with such abilities are the result of rational drug design and are termed antipathogenic drugs as opposed to antibacterial drugs (i.e., most traditional antibiotics). Antipathogenic drugs target key regulatory bacterial systems that govern the expression of virulence factors.

In recent years, researchers have come to appreciate that, in nature, most bacteria form complex surfaceattached (sessile) communities called biofilms. Bacteria present within biofilms have characteristics distinct from those of free-swimming (planktonic) bacteria of the same species, including a significantly increased tolerance to antimicrobial therapies and the host immune response (3). In modern clinical microbiology, the establishment of bacterial biofilms is often considered a pathogenicity trait during chronic infections (4). Biofilm formation is an example of microbial community behavior. Both Gram-positive and Gram-negative bacteria have been found to coordinate this behavior through cell-to-cell communication mediated by small, diffusible signals. This phenomenon has been termed quorum sensing and is prevalent among both symbiotic and pathogenic bacteria associated with plants and animals. Many of the phenotypes regulated by cell-to-cell communication are involved in bacterial colonization and virulence.

Among the Gram-negative bacteria, the most well studied quorum-sensing system is the LuxR-LuxI homologous system and the cognate signal molecules: $\mathrm{N}$-acyl-homoserine lactones (AHLs) (3). This quorumsensing system is widespread among Gram-negative genera and is involved in the regulation of many hostassociated phenotypes, including production of viru- 


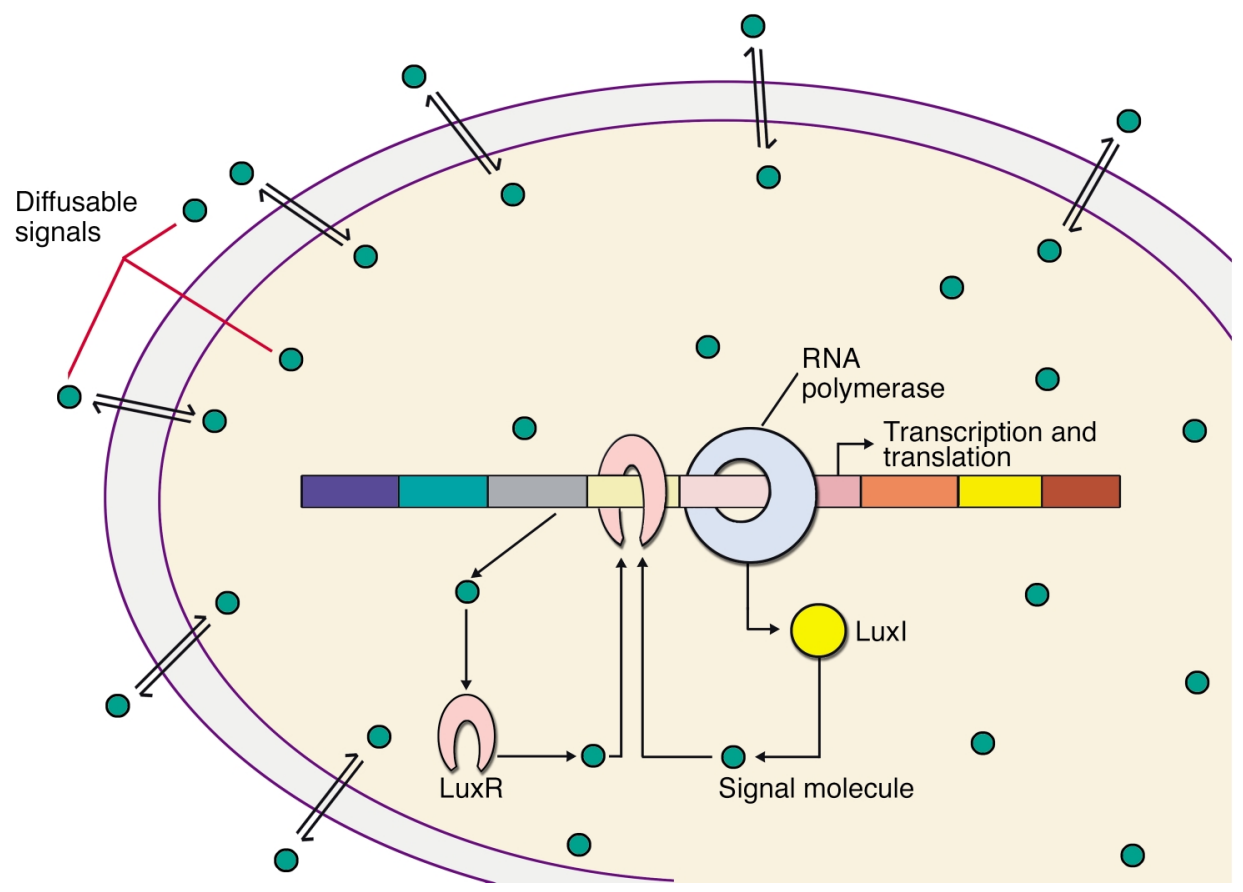

Figure 1

The archetypical Lux quorum sensor. The AHL signal (green circles) is synthesized by the luxl gene product Luxl (the synthase). At a certain threshold concentration, the AHL signal interacts with the receptor LuxR (encoded by luxR), which binds to the promoter sequence of the target genes (in this case, the lux operon) and in conjunction with the RNA polymerase promotes transcription.

lence factors (5-7) and secondary metabolites (8). Emerging evidence points to the involvement of quorum sensing in biofilm formation and surface motility in the opportunistic pathogens Pseudomonas aeruginosa (9), Burkbolderia cepacia (10), and Aeromonas bydrophila (11). These observations suggest that quorum sensing serves to link biofilm formation with virulence factor production. Interestingly, AHL-based cross-talk has been demonstrated between $P$. aeruginosa and $B$. cepacia (12) and between S. liquefaciens and P. aeruginosa (13).

The observation that quorum sensing is linked to virulence factor production and biofilm formation suggests that many virulent Gram-negative organisms could potentially be rendered nonpathogenic by inhibition of their quorum-sensing systems. Research into quorum sensing, and inhibition thereof, may provide a means of treating many common and damaging chronic infections without the use of growth-inhibitory agents, such as antibiotics, preservatives, and disinfectants, that unavoidably select for resistant organisms.

\section{AHL-mediated quorum sensing}

Quorum sensing is a generic regulatory mechanism used by many Gram-negative bacteria and Gram-positive bacteria to perceive and respond to factors as varied as changing microbial population density and the expression of specific genes. The concentration of a signal molecule reflects the density of bacterial cells in a defined environment, and the perception of a threshold level of that signal indicates that the population is "quorate," i.e., sufficiently dense to make a behavioral group-based decision.
Quorum sensing is thought to afford pathogenic bacteria a mechanism to minimize host immune responses by delaying the production of tissue-damaging virulence factors until sufficient bacteria have amassed and are prepared to overwhelm host defense mechanisms and establish infection. In our laboratory, we also view quorum sensing as a mechanism by which bacteria expose part of their genetic repertoire for recognition by other organisms (prokaryotes as well as eukaryotes): a phenomenon referred to as cross-talk. One environment that contains a large number of bacteria in close proximity is the bacterial biofilm. Furthermore, the dense and diffusion-limited biofilm matrix seems to provide ideal conditions for accumulation of signal molecules and a protected environment for bacteria to induce quorum sensing-regulated virulence factors and launch an attack on the host.

AHL-mediated quorum-sensing systems are found in a large number of Gram-negative bacterial species belonging to the $\alpha, \beta$, and $\gamma$ subclasses of proteobacteria, including bacteria in the genera Agrobacterium, Aeromonas, Burkholderia, Chromobacterium, Citrobacter, Enterobacter, Erwinia, Hafnia, Nitrosomonas, Obesumbacterium, Pantoea, Pseudomonas, Rabnella, Ralstonia, Rhodobacter, Rhizobium, Serratia, Vibrio, Xenorhabdus, and Yersinia (reviewed in ref. 3). The quorum-sensing system consists, in brief, of a four-component circuit: an AHL signal molecule, a LuxI-type signal synthase, a LuxRtype signal receptor, and the target gene(s) (Figure 1). The AHL signal is synthesized at a low basal level by the AHL synthase. AHL signals diffuse out of the bacteria and into the surrounding environment. An increase in 
a<smiles>[B]=CCCC(=O)N[C@H]1CCOC1=O</smiles><smiles>CCCCCCCCCCC(=O)CC(=O)NC1CCOC1=O</smiles>

b

C-30<smiles>O=C1C=C(Br)C(=CBr)O1</smiles>

C-56<smiles>O=C1C=CC(=CBr)O1</smiles><smiles>CCCCC1=C(Br)C(=CBr)OC1=O</smiles><smiles>CCCC(O)C1=C(Br)/C(=C/Br)OC1=O</smiles><smiles>CCCC(O)C1=C(Br)C(=CBr)OC1=O</smiles><smiles>CCCCC1=C(Br)C(=C(Br)Br)OC1=O</smiles>

d

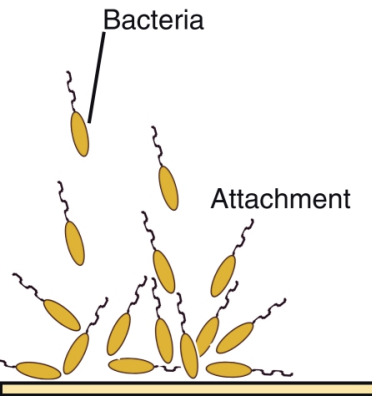
Early tower
structure
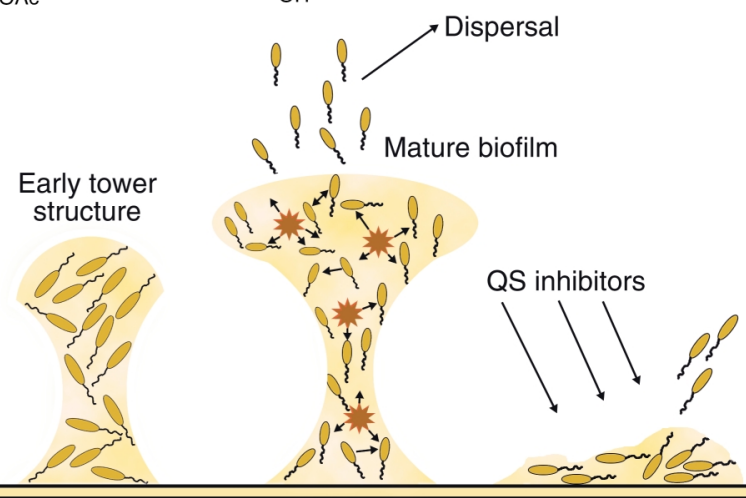

Dispersal O

\section{Figure 2}

(a) Molecular structures of the two cognate signal molecules produced by $P$. aeruginosa, BHL ([N-butyryl]-L-homoserine lactone), and OdDHL (N-[3-oxo-dodecanoyl]-L-homoserine lactone). (b) Synthetic quorum-sensing (QS) inhibitors derived from (c) natural brominated furanone compounds isolated from D. pulchra. (d) Temporal biofilm development and dispersal. Stars represent QS.

bacterial population density leads to an increase in local AHL concentration, and, at a threshold concentration, this signal interacts with a cognate receptor (LuxR-type response regulator) that in turn is activated as a positive transcription factor and modulates the expression of quorum sensing-regulated genes. Often, the quorum sensor is subject to autoinduction, because the gene encoding the signal synthase is among the target genes; hence, a positive feedback regulatory loop is created. The autoinduction allows a rapid increase in signal production and dissemination, which in turn induces a quorum sensing-controlled phenotype throughout the bacterial population (see Figure 1).

\section{Microbial biofilms and chronic infections}

Biofilms are now considered ubiquitous in the natural world (14). Bacterial biofilms have been observed to be extremely heterogeneous, both structurally and with regard to the physiology of the bacterial cells within them. The prevailing conceptual model depicts bacterial biofilms as being made up of microcolonies, which serve as the basic unit of the greater biofilm structure. Microcolonies are hydrated structures consisting of bacterial cells enmeshed in a matrix of exopolymeric substances (EPSs). Bacteria may proliferate on the attachment surface, leading to microcolony expansion. Eventually, community growth becomes limited by substrate availability due to increased diffusion distances, and the biofilm reaches a steady state. Such mature biofilms often consist of "towers" and "mushrooms" of cells in an EPS matrix (Figure 2). Interstitial voids and channels separate the biofilm structures and facilitate a convective flow in order to transport nutrients to interior parts of the biofilm and remove waste products. The biofilm mode of growth has been shown to facilitate bacterial survival in a variety of environmental stresses, including antibiotics and disinfectants $(4,15)$. Biofilms have become evident in many, if not most, environmental, industrial, and medical bacteriarelated problems. A recent public announcement from the NIH stated that more than $60 \%$ of all microbial infections involve biofilms (1).

\section{Quorum sensing as a target for antimicrobial therapy}

Given the many bacteria that employ quorum sensing in the control of virulence, quorum sensing constitutes a novel target for directed drug design (16). While AHLmediated quorum-sensing systems are employed by Gram-negative bacteria, many Gram-positive bacteria, 
including Bacillus subtilis, Streptococcus pneumoniae, and Staphylococcus aureus, use small peptides or modified peptides for signaling. Autoinducer 2 (AI-2), a signaling molecule common to many diverse bacteria, has recently been described (17). Genome sequencing revealed the presence of luxS homologs (encoding the AI-2 signal synthase) in many pathogens, including Escherichia coli, Helicobacter, Neisseria, Porphyromonas, Proteus, Salmonella, Enterococcus faecalis, Streptococcus pyogenes, and S. aureus (18). In some bacteria, AI-2 signaling is required for virulence (19), but in other bacteria, it does not appear essential for bacterial virulence (20). Recently, Winzer and colleagues suggested that, in most bacteria, AI-2 is simply a metabolic by-product and, therefore, doubtful as a drug target (21).

Quorum sensing-inhibitory compounds might constitute a new generation of antimicrobial agents with applications in many fields, including medicine (human and veterinary), agriculture, and aquaculture, and the associated commercial interests are substantial. Indeed, in recent years a number of biotechnology companies that aim specifically at developing antiquorum-sensing and anti-biofilm drugs have emerged (QSI Pharma A/S, Lyngby, Denmark; Microbia, Cambridge, Massachusetts, USA; Quorex Pharmaceuticals Inc., Carlsbad, California, USA; and 4SC AG, Martinsried, Germany). Several strategies aiming at the interruption of bacterial quorum-sensing circuits are possible, including (a) inhibition of AHL signal generation, (b) inhibition of AHL signal dissemination, and (c) inhibition of AHL signal reception.

\section{Inhibition of AHL signal generation}

The vast majority of bacteria that produce AHL signals encode one or more genes homologous to luxI of Vibrio fischeri (see Figure 1). Expression of these genes in heterologous host backgrounds has demonstrated that the LuxI-type protein is required and sufficient for production of AHL signals. The catalysis of AHL synthesis has been studied in vitro for three LuxI family members. The reaction involves a sequentially ordered reaction mechanism that uses $S$-adenosyl methionine (SAM) as the amino donor for generation of the homoserine lactone ring moiety, and an appropriately charged acyl carrier protein (ACP) as the precursor for the acyl side chain of the AHL signal (22).

Knowledge about signal generation can be exploited to develop quorum-sensing inhibitor molecules that target AHL signal generation. Various analogs of SAM, such as $S$-adenosylhomocysteine, $S$-adenosylcysteine, and sinefungin, have been demonstrated to be potent inhibitors of AHL synthesis catalyzed by the $P$. aeruginosa RhlI protein (22). The reaction chemistry of AHL synthase with SAM appears to be unique, even though SAM is a necessary and common intermediate in many prokaryotic and eukaryotic pathways. This raises the hope that SAM analogs could be used as specific inhibitors of quorum-sensing signal generation, without affecting eukaryotic enzymes that use SAM as a substrate. Some recent reports have demonstrated that certain macrolide antibiotics are capable of repressing $P$. aeruginosa AHL synthesis when applied at subminimal growth-inhibitory concentrations $(23,24)$. Erythromycin has been reported to suppress production of $P$. aeruginosa hemagglutinins, protease, hemolysin, and AHL signals (25). Macrolide antibiotics are generally recognized as inhibitors of protein synthesis at the ribosomal level. It remains unclear how these antibiotics interfere with quorum-sensing circuits. It is also unclear how resistance to these antibiotics affects their quorum sensing-modulatory properties.

\section{Inhibition of AHL signal dissemination}

Bacterial cell-to-cell communication can be inhibited by a decrease in the active signal-molecule concentration in the environment. AHL decay might be a consequence of a nonenzymatic reaction; e.g., AHL signals are subject to alkaline hydrolysis at high $\mathrm{pH}$ values (26). Some bacteria have been reported to specifically degrade AHL signals $(27,28)$. Dong et al. (27) found a Bacillus species that produced an enzyme, termed AiiA, that catalyzed the hydrolysis of AHL molecules. Expression of the aiiA gene in the plant pathogen Erwinia carotovora resulted in reduced release of AHL signals, decreased extracellular pectolytic enzyme activity, and attenuated soft rot disease symptoms in all plants tested (27). Moreover, transgenic plants expressing AiiA have been shown to be significantly less susceptible to infection by E. carotovora (29). In another study, a Variovorax paradoxus strain able to grow using 3-oxo-C6- $\mathrm{N}$ homoserine lactone as the sole energy and nitrogen source was isolated from a soil sample (28). In our own laboratory, we have conducted similar screenings and found that bacteria able to degrade or metabolize AHL molecules can be isolated from environmental samples at a high frequency (M. Givskov et al., unpublished observations). The ecological significance of such AHL-
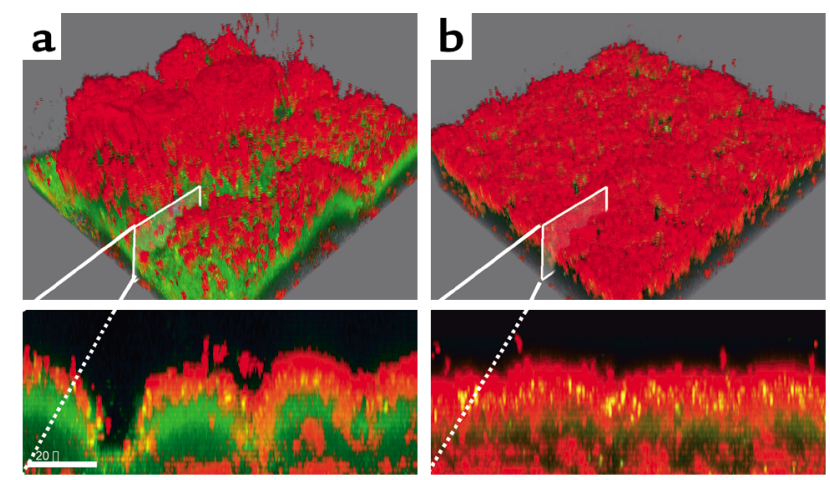

\section{Figure 3}

Furanone-treated $P$. aeruginosa biofilms are less tolerant to tobramycin. Scanning confocal laser photomicrographs of $P$. aeruginosa PAO1 biofilms grown in the absence $(\mathbf{a})$ or the presence $(\mathbf{b})$ of $10 \mu \mathrm{M} \mathrm{C}-30$. After 3 days, the biofilms were exposed to $100 \mu \mathrm{g} / \mathrm{ml}$ tobramycin for 24 hours. Bacterial viability was assayed by staining using the LIVE/DEAD BacLight Bacterial Viability Kit (Molecular Probes Inc., Eugene, Oregon, USA). Red areas are dead bacteria; green areas are live bacteria. 
degrading bacteria is not clear, but AHL-degrading enzymes are of great clinical interest for use in the prevention of diseases caused by quorum sensing-proficient bacterial populations.

\section{Inhibition of AHL signal reception}

Blocking of quorum-sensing signal transduction can be achieved by an antagonist molecule capable of competing or interfering with the native AHL signal for binding to the LuxR-type receptor. Competitive inhibitors would conceivably be structurally similar to the native AHL signal, in order to bind to and occupy the AHL-binding site but fail to activate the LuxR-type receptor. Noncompetitive inhibitors may show little or no structural similarity to AHL signals, as these molecules bind to different sites on the receptor protein.

Several reports describe the in vitro application of AHL analogs to achieve inhibition of the quorum-sensing circuits of various bacteria (30-34). These studies have generated substantial knowledge about the structure-function relationships of AHL signals, which is of great value for the continued search for potent quorum-sensing inhibitors. The acyl side chain has been modified in several ways, and it has been shown that the length is crucial to activity $(35,36)$. In one study of quorum sensing in E. carotovora, it was reported that increasing the length of the acyl side chain by one methylene unit reduced activity by $50 \%$, whereas a two-unit extension reduced activity by $90 \%$. Decreasing the chain length by one methylene unit decreased activity to $10 \%$ (35). Interestingly, AHL analogs with a longer side chain than the native AHL generally appear to be more efficient inhibitors than AHL analogs with a shorter side chain. This observation might suggest that a minimum acyl side chain length determined by the native AHL signal is required for binding to LuxR homologs and that longer acyl chains can be accommodated in the AHL-binding site of LuxR-type receptors. The flexibility of the acyl side chain also appears to be important for binding to LuxR-type proteins. For instance, reduction of the chain rotation by introduction of an unsaturated bond close to the amide linkage almost completely abolishes binding to the receptor $(31,34,35)$. In accordance with this suggestion, no natural AHL signal has ever been reported to contain a 2,3 unsaturated bond. A study investigating the P. aeruginosa LasR receptor suggested that the fully extended chain geometry is necessary for activation, whereas constrained analogs locked into different conformations showed no activity (37). The substitution at the $\beta$-position is important for the agonistic activity of AHLs, but there is no clear rule regarding the importance of this substitution to the maintenance of antagonistic activity.

The homoserine lactone moiety is generally very sensitive to modifications, and the chirality is crucial to biological activity. Natural AHL signals are L-isomers, whereas D-isomers are generally devoid of biological activity (35). The acyl side chain appears essential for activity, as exemplified in E. carotovora, in which the unsubstituted homoserine lactone ring fails to activate the quorum-sensing system (35). Conversion of the homoserine lactone ring to a homoserine lactame ring results in a molecule without agonistic or antagonistic properties $(30,35)$. Interestingly, a change of the homoserine lactone structure to a homoserine thiolactone ring appears permissible in several quorum-sensing systems $(30,31,35)$. A recent study showed that LasR and RhlR proteins responded differently to changes in the homoserine lactone moiety (38). This may indicate that the two $P$. aeruginosa AHL receptors differ significantly in their AHL-binding sites.

\section{Quorum-sensing inhibitors expressed by higher organisms}

A number of reports describe the ability of higher organisms to interfere with AHL-mediated quorum sensing. The best-characterized example is that of the Australian macroalga Delisea pulchra, described below. Recently, another example of eukaryotic interference with AHLmediated signaling was provided by Teplitski et al. (39), who showed that several plants secrete substances that mimic bacterial AHL signal activities and affect quorum sensing-regulated behaviors in associated bacteria. Exudates from pea (Pisum sativum) were demonstrated to contain several separable activities that either stimulated or inhibited bacterial AHL-dependent phenotypes (39). Many plants and fungi have coevolved and established carefully regulated symbiotic associations with bacteria. Interestingly, many plant-associated proteobacteria possess AHL-mediated quorum-sensing systems (40). Importantly, both plants and fungi are devoid of the active immune systems that are observed in mammals; rather, they rely on chemical defense systems to deal with bacteria in the environment. For these reasons, it might be expected that plants and fungi have evolved to produce chemical compounds to inhibit (or in other cases to stimulate) bacterial AHL-mediated communication. We have conducted screenings of plants (including some used in traditional herbal medicine) and fungal extracts for AHL-inhibitory activity (M. Givskov et al., unpublished results). A surprisingly large number of extracts contained quorum sensing-inhibitory activities. Not surprisingly, we found AHL-producing bacteria (which secrete hydrolytic exoenzymes) associated with these plants and their roots. We believe that the interplay of signals and signal inhibitors enables a stable coexistence of the eukaryotic host and the bacteria as long as the plant or root produces sufficient inhibitor to block the quorum-sensing systems of the colonizing organisms. Currently, work is in progress to characterize and isolate the pure compounds responsible for this quorum sensing-inhibitory activity.

\section{Inhibition of quorum sensing by halogenated furanone compounds}

The ability of bacteria to form biofilms is a major challenge for living organisms at risk of infection, such as humans, animals, and marine eukaryotes $(41,42)$. Marine plants are, in the absence of an advanced 
immune system, prone to disease $(43,44)$. Bacteria can be highly detrimental to marine algae and other eukaryotes (42). The Australian red macroalga D. pulchra produces a range of halogenated furanone compounds (45) that display antifouling and antimicrobial properties (46-48). This particular alga originally attracted the attention of marine biologists because it was devoid of surface colonization, i.e., biofouling, unlike other plants in the same environment. Biofouling is primarily caused by marine invertebrates and plants, but bacterial biofilms are believed to be the first colonizers of submerged surfaces, providing an initial conditioning biofilm to which other marine organisms may attach (49). Therefore, the abundance and composition of the bacterial community on the surface will significantly affect the subsequent development of a macrofouling community $(50,51)$. Consequently, eukaryotes have developed chemical defense mechanisms $(47,52,53)$ that, in several cases, include secondary metabolites that inhibit phenotypes relevant to bacterial colonization (54-56). Such secondary metabolites - furanones - are produced by the marine alga $D$. pulchra $(54,57,58)$.

$D$. pulchra furanone compounds consist in general of a furan ring structure with a substituted acyl chain at the $\mathrm{C}-3$ position and a bromine substitution at the $\mathrm{C}-4$ position (see Figure 2). The substitution at the C-5 position may vary in terms of side chain structure. The natural furanone is halogenated at various positions by bromine, iodide, or chloride (45). D. pulchra produces at least 30 different species of halogenated furanone compounds, which are stored in specialized vesicles and are released at the surface of the thallus at a concentration ranging from 1 to $100 \mathrm{ng} / \mathrm{cm}^{2}$. Field experiments have demonstrated that the surface concentration of furanones is inversely correlated to the degree of colonization by marine bacteria (55).

Givskov et al. (58) hypothesized that furanones of $D$. pulchra constitute a specific means of eukaryotic interference with bacterial signaling processes. An important discovery was the furanone-mediated displacement of radiolabeled AHL molecules from LuxR (59). This suggests that furanone compounds compete with the cognate AHL signal for the LuxR receptor site. Extensive experimental evidence in support of this model has accumulated during recent years. This includes the observations that furanones (a) repress AHL-dependent expression of $V$. fischeri bioluminescence (59); (b) inhibit AHL-controlled virulence factor production and pathogenesis in $P$. aeruginosa $(60,61)$; (c) inhibit quorum sensing-controlled luminescence and virulence of the black tiger prawn pathogen Vibrio harveyi (62); and, finally, (d) inhibit quorum sensing-controlled virulence of E. carotovora (63).

The natural furanone compounds have little or no effect on the quorum-sensing systems of $P$. aeruginosa. In collaboration with Staffan Kjelleberg's research group, we embarked on the process of drug development to find more potent quorum-sensing inhibitors. The natural furanone compounds were modified by chemical synthesis and screened for increased efficacy. Some derivatives of the $D$. pulchra furanone compounds were shown to repress quorum sensing in $P$. aeruginosa and reduce virulence factor expression $(60,61)$. Because synthetic compounds, which function well against planktonic cells, might be less efficient against biofilm bacteria, the efficacy of these quorum-sensing inhibitor compounds against bacterial biofilms was assayed. By means of AHL monitors built on the P. aeruginosa quorum sensors and the las $B-g f p$ target gene, the efficacy of these compounds was measured via GFP expression. The use of the GFP-based single-cell technology in combination with scanning confocal laser microscopy allowed estimation of furanone penetration and halflife and enabled us to identify synthetic compounds that not only inhibited the quorum sensors in the majority of the cells but also led to the formation of flat, undifferentiated biofilms that eventually detached (60). It is notable that the synthetic furanones, in concentrations that significantly lower quorum sensing-controlled gene expression in planktonic cells, were equally active against biofilm bacteria, despite the profoundly different modes of growth. In contrast, classical antibiotics used to treat $P$. aeruginosa infections, e.g., tobramycin and piperacillin, are required at concentrations 100- to 1,000-fold higher in order to kill biofilm bacteria than in order to kill their planktonic counterparts. In addition, we observed that furanone-treated biofilms were more susceptible to killing by tobramycin than their untreated counterparts (61) (Figure 3).

In a recently published report, we used DNA array technology to demonstrate that furanone compounds specifically repress expression of quorum sensing-controlled genes in $P$. aeruginosa (61). Microarray analysis of wildtype $P$. aeruginosa $\mathrm{PAO} 1$ showed that expression of 93 genes (1.7\% of the $P$. aeruginosa genome) was affected by the addition of the furanone compound C-30. Overall, 85 genes $(1.5 \%)$ were repressed and eight genes $(0.1 \%)$ were activated in response to $\mathrm{C}-30$. Genes encoding multidrug efflux pumps and transporters were predominant among the induced genes. The furanone-repressed genes included many previously known as quorum sensing-regulated genes, including numerous $P$. aeruginosa virulence factor genes such as las $B$ (encoding elastase), las $A$ (encoding LasA protease), $r b l A B$ operon (regulating rhamnolipid production), $p h z A-G$ operon (encoding phenazine biosynthesis), $h c n A B C$ operon (regulating hydrogen cyanide production), and chiC (encoding chitinase). To determine whether the remaining furanone-repressed genes were in fact controlled by quorum sensing, parallel mapping of the quorum-sensing regulon was performed using a las $I$ rblI double mutant grown with or without AHL signals. A comparative analysis showed that $80 \%$ of the furanonerepressed genes are indeed controlled by quorum sensing. Furthermore, furanone-repressed genes are not restricted to quorum sensing-regulated genes controlled by one of the two $P$. aeruginosa quorum-sensing circuits but may be controlled by either or both of the circuits (61). Microarray analysis demonstrated that expression of the 
lasI/lasR and $r b l I / r b l R$ gene clusters, which encode the central components of the $P$. aeruginos a quorum-sensing system, was not notably affected by furanone treatment. This observation suggests that the furanone does not interfere with some of the regulatory systems controlling transcription of the lasRI and rblRI genes, but rather that the furanone acts on these quorum-sensing regulators at the post-transcriptional level. Several genes involved in the biosynthesis of the Pseudomonas quinolone signal (PQS), including the $p h n A B$ operon and $p q s H$ (64), were also repressed by the furanone compound. Psendomonas quinolone signaling has been demonstrated, in concert with the AHL-based quorum-sensing systems, to be involved in the regulation of virulence factor production, in particular phenazine, pyocyanin, and hydrogen cyanide, and in autolysis of P. aeruginosa colonies (65).

A recent study has pointed at PQS signaling as an important regulatory function involved in $P$. aeruginosa adaptation and persistence in the cystic fibrotic lung environment (66). A pulmonary mouse infection model was used to study the effect of furanone compounds on the persistence of $P$. aeruginosa in chronic infections. Groups of mice infected with $P$. aeruginosa received subcutaneous furanone injections for 3 days, and this treatment was found to significantly reduce the bacterial load compared with that of the control group (67). Furthermore, the efficiency of bacterial clearing was positively correlated to the concentration of the furanone compound. The concentration used (as calculated by the whole-body concentration) was equal to or less than the concentrations required to inhibit expression of virulence factors in planktonic cultures and promote sloughing of in vitro biofilms.

\section{Discussion}

The purpose of research in this field has been to provide evidence that there are alternatives to the traditional mode of fighting bacterial infection. It is possible, from nature's own rich collection of chemical compounds, to generate powerful antipathogenic drugs that do not, per se, inhibit growth but instead interfere directly with microbial activity. The key to this concept is bacterial cellto-cell communication. Knowledge of the molecular mechanisms underlying these signaling systems and their control of virulence, biofilm formation, and pathogenicity brings a completely new perspective to the potential control of microbial activity. Current halogenated furanones are too reactive, and therefore presumably too toxic, for the treatment of bacterial infections in humans. However, their proven ability to control $P$. aeruginosa infections in animal models is of considerable importance, since it demonstrates that quorum sensing is a useful and promising drug target in vivo. On the other hand, several obvious disadvantages are associated with AHLbased quorum-sensing antagonists. First, each antagonist has a narrow spectrum, and, therefore, specific antagonists have to be developed for each organism targeted. This might, however, prove advantageous in some scenarios. For instance, it would theoretically be possible to attenuate a single, pathogenic organism living in a mixed population of normal bacterial flora by a specific inhibitor while leaving the rest of the bacterial population unaffected. Second, the therapeutic use of AHLbased antagonists is complicated by the fact that some AHL signal molecules function as virulence factors per se, as they possess immunomodulatory activities and affect muscle tissue as well as tracheal gland cells.

The ability to control $P$. aeruginosa with antipathogenic drugs holds great promise that a whole range of opportunistic, pathogenic bacteria can be controlled by similar pharmaceuticals. P. aeruginosa is an attractive model organism for such studies, partly because of the recent development of suitable cDNA microarray technology by Affymetrix Inc. (Santa Clara, California, USA). We have used this technique to demonstrate the target specificity of certain first-generation antipathogenic drugs (61). We envision that this approach will be used in many primary research and pharmaceutical laboratories in the future quest for drugs that target specific cellular components or interactions. Given the large number of bacteria that employ quorum-sensing communication systems, chemical attenuation of unwanted bacterial activities rather than bactericidal or bacteriostatic strategies may find application in many different fields, e.g., in medicine, agriculture, and food technology. This new concept is highly attractive because it is unlikely to pose a selective pressure for the development of resistance. The present approach is therefore generic in nature and highly promising for defense against bacterial biofilms encountered in many infectious diseases, on medical implants, and in many industrial facilities and water pipelines.

\section{Acknowledgments}

The authors wish to acknowledge financial support from the Danish Technical Research Council, the Danish Medical Research Council, the Plasmid Foundation, the Villum Kann Rasmussen Foundation, and Cystic Fibrosis Foundation Therapeutics Inc.

1. Lewis, K. 2001. Riddle of biofilm resistance. Antimicrob. Agents Chemother. 45:999-1007.

2. Hancock, R.E. 1998. Resistance mechanisms in Pseudomonas aeruginosa and other nonfermentative gram-negative bacteria. Clin. Infect. Dis. 27(Suppl. 1):S93-S99.

3. Eberl, L. 1999. $N$-acyl homoserinelactone-mediated gene regulation in gram-negative bacteria. Syst. Appl. Microbiol. 22:493-506.

4. Costerton, J.W., Stewart, P.S., and Greenberg, E.P. 1999. Bacterial biofilms: a common cause of persistent infections. Science. 284:1318-1322.

5. Passador, L., Cook, J.M., Gambello, M.J., Rust, L., and Iglewski, B.H. 1993. Expression of Pseudomonas aeruginosa virulence genes requires cellto-cell communication. Science. 260:1127-1130.

6. von Bodman, S.B., and Farrand, S.K. 1995. Capsular polysaccharide biosynthesis and pathogenicity in Erwinia stewartii require induction by an $N$-acylhomoserine lactone autoinducer. J. Bacteriol. 177:5000-5008.

7. Lewenza, S., Conway, B., Greenberg, E.P., and Sokol, P.A. 1999. Quorum sensing in Burkholderia cepacia: identification of the LuxRI homologs CepRI. J. Bacteriol. 181:748-756.

8. Latifi, A., et al. 1995. Multiple homologues of LuxR and LuxI control expression of virulence determinants and secondary metabolites through quorum sensing in Pseudomonas aeruginosa PAO1. Mol. Microbiol. 17:333-343.

9. Davies, D.G., et al. 1998. The involvement of cell-to-cell signals in the development of a bacterial biofilm. Science. 280:295-298. 
10. Huber, B., et al. 2001. The cep quorum-sensing system of Burkholderia cepacia H111 controls biofilm formation and swarming motility. Microbiology. 147:2517-2528.

11. Lynch, M.J., et al. 2002. The regulation of biofilm development by quorum sensing in Aeromonas hydrophila. Environ. Microbiol. 4:18-28.

12. Riedel, K., et al. 2001. $N$-acylhomoserine-lactone-mediated communication between Pseudomonas aeruginosa and Burkholderia cepacia in mixed biofilms. Microbiology. 147:3249-3262.

13. Rasmussen,T.B., et al. 2000. How Delisea pulchra furanones affect quorum sensing and swarming motility in Serratia liquefaciens MG1. Microbiology. 146:3237-3244

14. Costerton, J.W., et al. 1987. Bacterial biofilms in nature and disease. Annu. Rev. Microbiol. 41:435-464.

15. Xu, K.D., McFeters, G.A., and Stewart, P.S. 2000. Biofilm resistance to antimicrobial agents. Microbiology. 146:547-549.

16. Hartman, G., and Wise, R. 1998. Quorum sensing: potential means of treating gram-negative infections? Lancet. 351:848-849.

17. Chen, X., et al. 2002. Structural identification of a bacterial quorumsensing signal containing boron. Nature. 415:545-549.

18. Xavier, K.B., and Bassler, B.L. 2003. LuxS quorum sensing: more than just a numbers game. Curr. Opin. Microbiol. 6:191-197.

19. Soo, Y.K., et al. 2003. Regulation of Vibrio vulnificus virulence by the LuxS quorum-sensing system. Mol. Microbiol. 48:1647-1664.

20. Hubner, A., Revel, A.T., Nolen, D.M., Hagman, K.E., and Norgard, M.V 2003. Expression of a $\operatorname{luxS}$ gene is not required for Borrelia burgdorferi infection of mice via needle inoculation. Infect. Immun. 71:2892-2896.

21. Winzer, K., et al. 2002. LuxS: its role in central metabolism and the in vitro synthesis of 4-hydroxy-5-methyl-3(2H)-furanone. Microbiology. 148:909-922.

22. Parsek, M.R., Val, D.L., Hanzelka, B.L., Cronan, J.E., Jr., and Greenberg, E.P. 1999. Acyl homoserine-lactone quorum-sensing signal generation. Proc. Natl. Acad. Sci. U. S. A. 96:4360-4365.

23. Tateda, K., et al. 2001. Azithromycin inhibits quorum sensing in Pseudomonas aeruginosa. Antimicrob. Agents Chemother. 45:1930-1933.

24. Pechere, J.C. 2001. Azithromycin reduces the production of virulence factors in Pseudomonas aeruginosa by inhibiting quorum sensing. Jpn.J. Antibiot. 54:87-89.

25. Sofer, D., Gilboa-Garber, N., Belz, A., and Garber, N.C. 1999. 'Subinhibitory' erythromycin represses production of Pseudomonas aeruginosa lectins, autoinducer and virulence factors. Chemotherapy. 45:335-341.

26. Yates, E.A., et al. 2002. N-acylhomoserine lactones undergo lactonolysis in a $\mathrm{pH}$-, temperature-, and acyl chain length-dependent manner during growth of Yersinia pseudotuberculosis and Pseudomonas aeruginosa. Infect. Immun. 70:5635-5646.

27. Dong, Y.H., Xu, J.L., Li, X.Z., and Zhang, L.H. 2000. AiiA, an enzyme that inactivates the acylhomoserine lactone quorum-sensing signal and attenuates the virulence of Erwinia carotovora. Proc. Natl. Acad. Sci. U. S. A. 97:3526-3531.

28. Leadbetter, J.R., and Greenberg, E.P. 2000. Metabolism of acyl-homoserine lactone quorum-sensing signals by Variovorax paradoxus. J. Bacteriol. 182:6921-6926.

29. Dong, Y.H., et al. 2001. Quenching quorum-sensing-dependent bacterial infection by an N-acyl homoserine lactonase. Nature. 411:813-817.

30. Passador, L., et al. 1996. Functional analysis of the Pseudomonas aeruginosa autoinducer PAI. J. Bacteriol. 178:5995-6000.

31. Schaefer, A.L., Hanzelka, B.L., Eberhard, A., and Greenberg, E.P. 1996 Quorum sensing in Vibrio fischeri: probing autoinducer-LuxR interactions with autoinducer analogs. J. Bacteriol. 178:2897-2901.

32. Swift, S., et al. 1997. Quorum sensing in Aeromonas bydrophila and Aeromonas salmonicida: identification of the LuxRI homologs AhyRI and AsaRI and their cognate $N$-acylhomoserine lactone signal molecules. J. Bacteriol. 179:5271-5281.

33. Swift, S., et al. 1999. Quorum sensing-dependent regulation and blockade of exoprotease production in Aeromonas hydrophila. Infect. Immun. 67:5192-5199.

34. Zhu, J., et al. 1998. Analogs of the autoinducer 3-oxo-octanoyl-homoserine lactone strongly inhibit activity of the TraR protein of Agrobacterium tumefaciens. J. Bacteriol. 180:5398-5405.

35. Chhabra, S.R., et al. 1993. Autoregulation of carbapenem biosynthesis in Erwinia carotovora by analogues of $\mathrm{N}$-(3-oxohexanoyl)-L-homoserine lactone. J. Antibiot. (Tokyo.) 46:441-454.

36. McClean, K.H., et al. 1997. Quorum sensing and Chromobacterium violaceum: exploitation of violacein production and inhibition for the detection of $N$-acylhomoserine lactones. Microbiology. 143:3703-3711.

37. Kline, T., et al. 1999. Novel synthetic analogs of the Pseudomonas autoinducer. Bioorg. Med. Chem. Lett. 9:3447-3452.

38. Smith, K.M., Bu, Y., and Suga, H. 2003. Induction and inhibition of Pseudomonas aeruginosa quorum sensing by synthetic autoinducer analogs. Chem. Biol. 10:81-89.

39. Teplitski, M., Robinson, J.B., and Bauer, W.D. 2000. Plants secrete substances that mimic bacterial $N$-acyl homoserine lactone signal activities and affect population density-dependent behaviors in associated bacteria. Mol. Plant Microbe Interact. 13:637-648.

40. Cha, C., Gao, P., Chen, Y.C., Shaw, P.D., and Farrand, S.K. 1998. Production of acyl-homoserine lactone quorum-sensing signals by gram-negative plant-associated bacteria. Mol. Plant Microbe Interact. 11:1119-1129.

41. Kushmaro, A., Loya, Y., Fine, E., and Rosenberg, E. 1996. Bacterial infection and coral bleaching. Nature. 380:396.

42. Littler, M.M., and Littler, D.S. 1995. Impact of CLOD pathogen on Pacific coral reefs. Science. 267:1356-1360.

43. Correa, J.A. 1996. Diseases in seaweeds: an introduction. Hydrobiologia. 326:87-88.

44. Fenical, W. 1997. New pharmaceuticals from marine organisms. Trends Biotechnol. 15:339-341.

45. de Nys, R., Wright, A.D., König, G.M., and Sticher, O. 1993. New halogenated furanones from the marine alga Delisea pulchra. Tetrahedron. 49:11213-11220

46. de Nys, R., Steinberg, P., Rogers, C.N., Charlton, T.S., and Duncan, M.W. 1996. Quantitative variation of secondary metabolites in the sea hare Apylsia parvula and its host plant, Delisea pulchra. Mar. Ecol. Prog. Ser. 130:135-146.

47. de Nys, R., et al. 1995. Broad spectrum effects of secondary metabolites from the red alga Delisea pulchra in antifouling assays. Biofouling. 8:259-271.

48. Reichelt, J.L., and Borowitzka, M.A. 1984. Antimicrobial activity from marine algae: results of a large-scale screening programme. Hydrobiology. 116/117:158-168.

49. Rice, S.A., Givskov, M., Steinberg, P., and Kjelleberg, S. 1999. Bacterial signals and antagonists: the interaction between bacteria and higher organisms. J. Mol. Microbiol. Biotechnol. 1:23-31.

50. Belas, M.R. 2003. The swarming phenomenon of Proteus mirabilis. ASM News. 58:15-22.

51. Henschel, J.R., and Cook, P.A. 1990. The development of a marine fouling community in relation to the primary film of microorganisms. Biofouling. 2:1-11.

52. Davis, A.R., et al. 1989. Epibiosis of marine algae and benthic invertebrates: natural products chemistry and other mechanisms inhibiting settlement and overgrowth. Bioorganic Marine Chemistry. 3:86-114.

53. Wahl, M. 2003. Marine epibiosis. Fouling and antifouling: some basic aspects. Mar. Ecol. Prog. Ser. 58:175-189.

54. Kjelleberg, S., et al. 1997. Do marine natural products interfere with prokaryotic AHL regulatory systems? Aquat. Microb. Ecol. 13:85-93.

55. Maximilien, R.R., et al. 1998. Chemical mediation of bacterial surface colonisation by secondary metabolites from the red alga Delisea pulchra. Aquat. Microb. Ecol. 15:233-246.

56. Slattery, M., McClintoch, J.B., and Heine, J.N. 1995. Chemical defences in Antarctic soft corals: evidence for antifouling compounds. J. Exp. Mar. Biol. Ecol. 190:61-77.

57. de Nys, R., et al. 1995. Broad spectrum effects of secondary metabolites from the red alga Delisea pulchra in antifouling assays. Biofouling. 8:259-271.

58. Givskov, M., et al. 1996. Eukaryotic interference with homoserine lactone-mediated prokaryotic signalling. J. Bacteriol. 178:6618-6622.

59. Manefield, M., et al. 1999. Evidence that halogenated furanones from Delisea pulchra inhibit acylated homoserine lactone (AHL)-mediated gene expression by displacing the AHL signal from its receptor protein. Microbiology. 145:283-291.

60. Hentzer, M., et al. 2002. Inhibition of quorum sensing in Pseudomonas aeruginosa biofilm bacteria by a halogenated furanone compound. Microbiology. 148:87-102.

61. Hentzer, M., et al. 2003. Attenuation of Pseudomonas aeruginosa virulence by quorum sensing inhibitors. EMBOJ. 22:3803-3815.

62. Manefield, M., Harris, L., Rice, S.A., de Nys, R., and Kjelleberg, S. 2000. Inhibition of luminescence and virulence in the black tiger prawn (Penaeus monodon) pathogen Vibrio harveyi by intercellular signal antagonists. Appl. Environ. Microbiol. 66:2079-2084.

63. Manefield, M., Welch, M., Givskov, M., Salmond, G.P., and Kjelleberg, S. 2001. Halogenated furanones from the red alga, Delisea pulchra, inhibit carbapenem antibiotic synthesis and exoenzyme virulence factor production in the phytopathogen Erwinia carotovora. FEMS Microbiol. Lett. 205:131-138.

64. Gallagher, L.A., McKnight, S.L., Kuznetsova, M.S., Pesci, E.C., and Manoil, C. 2002. Functions required for extracellular quinolone signaling by Pseudomonas aeruginosa. J. Bacteriol. 184:6472-6480.

65. D’Argenio, D.A., Calfee, M.W., Rainey, P.B., and Pesci, E.C. 2002. Autolysis and autoaggregation in Pseudomonas aeruginosa colony morphology mutants. J. Bacteriol. 184:6481-6489.

66. Guina, T., et al. 2003. Quantitative proteomic analysis indicates increased synthesis of a quinolone by Pseudomonas aeruginosa isolates from cystic fibrosis airways. Proc. Natl. Acad. Sci. U. S. A. 100:2771-2776.

67. Wu, H., et al. 2000. Detection of $N$-acylhomoserine lactones in lung tissues of mice infected with Pseudomonas aeruginosa. Microbiology. 146:2481-2493. 